Pacific Journal of Mathematics

EXISTENCE THEOREMS FOR CERTAIN CLASSES OF
TWO-POINT BOUNDARY PROBLEMS BY VARIATIONAL

RICHARD J. DRISCOL 


\title{
EXISTENCE THEOREMS FOR CERTAIN CLASSES OF TWO-POINT BOUNDARY PROBLEMS BY VARIATIONAL METHODS
}

\author{
RICHARD J. DRISCOLL
}

Prefatory remarks. The principal results of this paper are existence theorems for solutions of two classes of vector differential systems; in each case the existence theorem is established by variational methods. In particular, the second system considered is a generalization of a scalar system, including as a special case the so-called Fermi-Thomas equation, studied by Sansone [8; pp. 445-450]. In spite of similarities occurring in the discussion of the two systems considered, the two problems are sufficiently distinct to warrant separate treatment. Accordingly, we shall divide the remaining sections of this paper into two parts, in which the numbering of sections and of displayed material will be independent; the bibliography, however, will apply to both parts.

Matrix notation will be used throughout and all matrices will have real elements; in particular, a vector $u=\left(u_{j}\right),(j=1,2, \cdots, n)$, will be regarded as an $n \times 1$ matrix. If $M$ is a matrix, $M^{*}$ will denote the transpose of $M$, while for a vector $u=\left(u_{j}\right),(j=1,2, \cdots, n)$, we define $|u|=\left(u_{1}^{2}+\cdots+u_{n}^{2}\right)^{1 / 2}$. For $F(u)$ a scalar function of the vector $u$, the symbol $F_{u}(u)$ will denote the vector function $\left(F_{u_{j}}(u)\right)$; if $G(u)$ is a vector function $\left(G_{i}(u)\right),(i=1,2, \cdots, m)$, of the vector $u$, then $G_{u}(u)$ will denote the $m \times n$ matrix $\left\|\partial G_{i} \mid \partial u_{j}\right\|,(i=1, \cdots, m ; j=1, \cdots, n)$. If $M$ and $N$ are matrices, the notation $M \geq N$ is used to signify that $M$ and $N$ are real symmetric matrices of the same dimensions and $M-N$ is nonnegative. As usual, the symbol $C^{(n)}$ represents the class of finite dimensional matrix functions which are continuous and have continuous derivatives of the first $n$ orders on some given set.

\section{PART I}

1. Introduction. This part of the paper will be concerned with vector differential systems of the form

$$
\begin{aligned}
y^{\prime \prime} & =f\left(x, y, y^{\prime}\right), & a \leq x \leq b, \\
y(a) & =y_{1}, \quad y(b)=y_{2}, &
\end{aligned}
$$

Received March 2, 1959. 1 This research was supported in part by the United States Air Force through the Air Force Office of Scientific Research of the Air Research and Development Command, under contract Nos. AF-(603)-86 and AF-49(638)-400. Reproduction in whole or in part is permitted for any purpose of the United States Government This paper was part of a thesis submitted to Northwestern University in partial fulfillment of the requirements for the degree of Doctor of Philosophy. The author wishes to express his gratitude to Professor W. T. Reid for his encouragement and guidance. 
where $f(x, y, z)$ is an $n$-dimensional real vector function of the real scalar $x$ and the real $n$-dimensional vectors $y$ and $z$. It will be shown that the system (1.1) has a solution, under the hypotheses $H_{1}, H_{2}, H_{3}, H_{5}$, of $\S 3$ and $H_{4}^{*}$ of $\S 4$. For reasons of convenience, we shall work primarily with the system

$$
\begin{aligned}
y^{\prime \prime} & =f\left(x, y, y^{\prime}\right), & a \leq x \leq b, \\
y(a) & =0=y(b), &
\end{aligned}
$$

and show in $\S 4$ how a system (1.1) may be reduced to such a system. The existence proof will use variational methods applied to the functional

$$
I(y, z) \equiv \int_{a}^{b}\left(\left|y^{\prime}-z\right|^{2}+\left|z^{\prime}-f(x, y, z)\right|^{2}\right) d x,
$$

with $(y, z)$ in the class $K$ of function pairs defined below. In $\S 2$ there are listed some lemmas to be used later. In $\S 3$ an existence theorem for a solution of (1.2) is established by showing, in effect, that $I(y, z)$ has a minimum for $(y, z)$ in $K$, and that this minimum is zero. The relation between systems of the forms (1.1) and (1.2) is considered in $\S 4$, while $\S 5$ contains a comment on a modification of hypotheses. Finally, $\S 6$ is devoted to an example of a class of problems to which the existence theorem proved here is applicable.

In what follows, $A_{2}$ will denote the class of vector functions $y(x)$ which are absolutely continuous and for which $\left|y^{\prime}(x)\right|^{2}$ is integrable on $a \leq x \leq b$, while $K$ is the class of vector function pairs $(y, z)$ with $y(x)$ and $z(x)$ in $A_{2}$ and with $y(a)=0=y(b)$.

2. Some useful lemmas. For future reference we collect here certain auxiliary results.

Lemma 2.1. Suppose that the matrix $f_{z}(x, y, z)$ exists and is continuous for $a \leq x \leq b$, all $y$, and all $z$. If for each $\rho>0$ the elements of $f_{z}$ are bounded for $a \leq x \leq b,|y|<\rho$ and $z$ arbitrary, then there are values $K_{1}=K_{1}(\rho)$ and $K_{2}=K_{2}(\rho)$ such that

$$
|f(x, y, z)| \leq K_{1}|z|+K_{2}, \text { for } a \leq x \leq b,|y|<\rho, z \text { arbitrary. }
$$

Lemma 2.2. If $\left\{w_{m}(x)\right\},(m=1,2, \cdots)$, is a sequence of vector functions of class $A_{2}$ such that the two sequences $\left\{\int_{a}^{b}\left|w_{m}\right|^{2} d x\right\}$ and $\left\{\int_{a}^{b}\left|w_{m}^{\prime}\right|^{2} d x\right\}$ are bounded, then the $w_{m}(x)$ are uniformly bounded on $a \leq x \leq b$, and there exists $a w(x)$ in $A_{2}$ and a subsequence $\left\{w_{m_{j}}(x)\right\}$ such that $w_{m_{j}}(x) \rightarrow w(x)$ uniformly and $w_{m_{j}}^{\prime}(x) \rightarrow w^{\prime}(x)$ weakly in the class of integrable square functions on $a \leq x \leq b$. 
This lemma is a ready consequence of well-known results for the Hilbert space of real-valued measurable functions whose squares are Lebesgue integrable on $a \leq x \leq b$, see, for example, [7; $\S 32,99$ ].

LEMMA 2.3. If $y(x)$ is in $A_{2}$ and $y(a)=0$, then

$$
\int_{a}^{b}\left|y^{\prime}\right|^{2} d x \geq \frac{\pi^{2}}{4(b-a)^{2}} \int_{a}^{b}|y|^{2} d x
$$

This is a well-known condition on the smallest proper value of the differential system $y^{\prime \prime}+\lambda y=0, y(a)=0=y^{\prime}(b)$. For an independent proof see [2; p. 184]; the present inequality follows from (7.7.1) of [2] by a simple change of variable.

We will also need some results related to non-oscillation of the scalar differential equation

$$
\left(\psi_{1}(x) u^{\prime}(x)\right)^{\prime}-\psi_{2}(x) u(x)=0, \quad a \leq x \leq b,
$$

where $\psi_{1}$ is of class $C^{\prime}$ and $\psi_{2}$ continuous on $a \leq x \leq b$. The equation (2.1) is termed non-oscillatory on $a \leq x \leq b$ if for two arbitrary points $x_{1}, x_{2}$ satisfying $a \leq x_{1}<x_{2} \leq b$, any solution $u(x)$ of (2.1) vanishing at $x_{1}$ and at $x_{2}$ vanishes identically on $a \leq x \leq b$. It is well-known that if $\psi_{1}(x)>0$ on $a \leq x \leq b$, then (2.1) is non-oscillatory on $a \leq x \leq b$ if and only if

$$
J(u) \equiv \int_{a}^{b}\left(\psi_{1}(x) u^{\prime 2}(x)+\psi_{2}(x) u^{2}(x)\right) d x>0
$$

holds for all non-identically vanishing $u(x)$ belonging to $A_{2}$ and satisfying $u(a)=0=u(b)$. For a proof of this statement see, for example, [5; Theorem 2.1], where a more general result is proved. Moreover, if (2.1) is non-oscillatory on $a \leq x \leq b$, the infimum of $J(u)$ for $u(x)$ in $A_{2}$ and satisfying $u(a)=0=u(b), \int_{a}^{b} u^{2} d x=1$ is greater than zero, as can be seen from an indirect argument. Indeed, if the infimum were equal to zero, then there would be a sequence of functions $u_{j}$ in $A_{2}$ with $u_{j}(a)=$ $0=u_{j}(b), \int_{a}^{b} u_{j}^{2} d x=1, j=1,2, \cdots$, and with $J\left(u_{j}\right) \rightarrow 0$. One readily verifies that the sequence $\left\{\int_{a}^{b} u_{j}^{\prime 2} d x\right\}$ would be bounded, so that, by Lemma 2.2, there would be a $u(x)$ in $A_{2}$ and a subsequence of $\left\{u_{j}\right\}$, denoted again by $\left\{u_{j}\right\}$, such that $u_{j}(x) \rightarrow u(x)$ uniformly on $a \leq x \leq b$, and $u_{j}^{\prime}(x)$ $\rightarrow u^{\prime}(x)$ weakly on this interval. The identity

$$
J\left(u_{j}\right)=J(u)+\int_{a}^{b} \psi_{1}(x)\left[2 u^{\prime}\left(u_{j}^{\prime}-u^{\prime}\right)+\left(u_{j}^{\prime}-u^{\prime 2}\right)\right] d x+\int_{a}^{b} \psi_{2}(x)\left(u_{j}^{2}-u^{2}\right) d x
$$

would then imply that $0=\lim J\left(u_{j}\right) \geq J(u)$, contrary to (2.2), since $u(a)=0=u(b)$ and $\int_{a}^{b} u^{2} d x=1$. With these comments one readily establishes the following result. 
LEMMA 2.4. If (2.1) is non-oscillatory on $a \leq x \leq b$, and $\psi_{1}(x)>0$ on this interval, then there exists an $\varepsilon>0$ such that if $h(x)$ is any function continuous and satisfying $|h(x)|<\varepsilon$ on $a \leq x \leq b$, then the equation $\left(\psi_{1}(x) u^{\prime}\right)^{\prime}-\left(\psi_{2}(x)+h(x)\right) u=0$ is non-oscillatory on $a \leq x \leq b$.

3. Existence theorem for a solution of (1.2). In the future sections we will make reference to the following hypotheses on the real-valued vector function $f(x, y, z)$ :

$H_{1} . \quad f(x, y, z)$ is continuous for $(x, y, z)$ in $\Omega: a \leq x \leq b,|y|<\infty$, $|z|<\infty$. in $\Omega$.

$H_{2}$. The matrices $f_{y}$ and $f_{z}$ exist and are continuous for $(x, y, z)$

$H_{3}$. For any $\rho>0$, there exists a $K=K_{\rho}$ such that $\left|\partial f_{i}\right| \partial z_{j} \mid \leq K$ for $|y|<\rho, a \leq x \leq b,|z|<\infty,(i, j=1, \cdots, n)$.

$H_{4}$. For arbitrary $\rho>0$ there exist scalar functions $\psi_{1}(x)=$ $\psi_{1}(x ; \rho) \in C, \psi_{2}(x)=\psi_{2}(x ; \rho) \in C^{\prime}$ with $\psi_{2}(x)>0$ on $a \leq x \leq b$, and $a$ constant $N=N(\rho)$ such that:

(a) the scalar differential equation $\left(\psi_{2}(x) w^{\prime}\right)^{\prime}-\psi_{1}(x) w=0$ is nonoscillatory on $a \leq x \leq b$;

(b) the integral inequality

$$
2 \int_{a}^{b} y^{*} f(x, y, z) d x \geq \int_{a}^{b}\left[\left(\psi_{2}-1\right)\left|y^{\prime}\right|^{2}+\psi_{1}|y|^{2}\right] d x-N
$$

holds for all $y(x), z(x)$ in $A_{2}$ satisfying $y(a)=0=y(b)$ and

$$
\int_{a}^{b}\left|y^{\prime}-z\right|^{2} d x \leq \rho .
$$

$H_{5}$. For arbitrary $y(x), z(x)$ in $A_{2}$, the vector differential system

$$
\begin{gathered}
w^{\prime \prime}-f_{z}(x, y(x), z(x)) w^{\prime}-f_{y}(x, y(x), z(x)) w=0, \quad a \leq x \leq b \\
w(a)=0=w(b)
\end{gathered}
$$

has only the solution $w(x) \equiv 0, a \leq x \leq b$.

We now prove the following theorem.

THEOREM 3.1. Under the hypotheses $H_{1}-H_{5}$ there exists a solution of the system (1.2).

Let $\left\{y_{m}(x), z_{m}(x)\right\}, m=1,2, \cdots$, be a sequence of function pairs of class $K$ such that $I\left(y_{m}, z_{m}\right) \rightarrow I_{0}$, where $I_{0}$ denotes the infimum of $I(y, z)$ on $K$. Since $\left\{I\left(y_{m}, z_{m}\right)\right\}$ is a convergent sequence, there exists a constant $M_{0}$ such that $I\left(y_{m}, z_{m}\right) \leq M_{0}, m=1,2, \cdots$. It will be shown first that the inequality

$$
\int_{a}^{b}\left(\left|y_{m}^{\prime}(x)\right|^{2}+2 y_{m}^{*}(x) f\left(x, y_{m}(x), z_{m}(x)\right)\right) d x \leq M, \quad m=1,2, \cdots
$$


holds for

$$
M=2 M_{0} / k, \text { where } k=\operatorname{Min}\left(1, \pi^{2} /\left[4(b-a)^{2}\right]\right) .
$$

Let $v_{m}(x)=\int_{a}^{x} f_{m}(s) d s+z_{m}(a)$, where $f_{m}(x)=f\left(x, y_{m}(x), z_{m}(x)\right)$. Then $u_{m}(x)=z_{m}(x)-v_{m}(x)$ is in $A_{2}$, and $u_{m}(a)=0$, so that by Lemma 2.3,

$$
\int_{a}^{b}\left|z_{m}^{\prime}-f_{m}\right|^{2} d x=\int_{a}^{b}\left|u_{m}^{\prime}\right|^{2} d x \geq \frac{\pi^{2}}{4(b-a)^{2}} \int_{a}^{b}\left|u_{m}\right|^{2} d x .
$$

This inequality yields

$$
M_{0} \geq k \int_{a}^{b}\left(\left|y_{m}^{\prime}-z_{m}\right|^{2}+\left|z_{m}-v_{m}\right|^{2}\right) d x \geq(k / 2) \int_{a}^{b}\left|y_{m}^{\prime}-v_{m}\right|^{2} d x,
$$

where $k$ is as in (3.3). Since

$$
\int_{a}^{b} y_{m}^{\prime *} v_{m} d x=\left.y_{m}^{*} v_{m}\right|_{a} ^{b}-\int_{a}^{b} y_{m}^{*} v_{m}^{\prime} d x=-\int_{a}^{b} y_{m}^{*} f_{m} d x,
$$

relation (3.2), with $M$ given by (3.3), results from (3.4) and the obvious inequality

$$
\int_{a}^{b}\left|y_{m}^{\prime}-v_{m}\right|^{2} d x \geq \int_{a}^{b}\left(\left|y_{m}^{\prime}\right|^{2}-2 y_{m}^{* \prime} v_{m}\right) d x
$$
write

Since the sequence $\left\{\int_{a}^{b}\left|y_{m}^{\prime}-z_{m}\right|^{2} d x\right\}$ is bounded, we may use $H_{4}$ to

$$
2 \int_{a}^{b} y_{m}^{*} f\left(x, y_{m}, z_{m}\right) d x \geq \int_{a}^{b}\left[\left(\psi_{2}-1\right)\left|y_{m}^{\prime}\right|^{2}+\psi_{1}\left|y_{m}\right|^{2}\right] d x-N,
$$

where $\psi_{2}(x), \psi_{1}(x)$, and $N$ have the properties stated in $H_{4}$. This relation with (3.2) yields

$$
\int_{a}^{b}\left(\psi_{2}\left|y_{m}^{\prime}\right|^{2}+\psi_{1}\left|y_{m}\right|^{2}\right) d x \leq M+N
$$

Since $\left(\psi_{2}(x) u^{\prime}\right)^{\prime}-\psi_{1}(x) u=0$ is non-oscillatory on $a \leq x \leq b$, Lemma 2.4 implies that there is an $r$ with $0<r<1$ such that $\left(\psi_{2} u^{\prime}\right)^{\prime}-(1 / r) \psi_{1} u=0$ is non-oscillatory on $a \leq x \leq b$. As $y_{m}(a)=0=y_{m}(b)$, we then have

$$
\int_{a}^{b}\left(r \psi_{2}\left|y_{m}^{\prime}\right|^{2}+\psi_{1}\left|y_{m}\right|^{2}\right) d x \geq 0
$$

and

$$
\int_{a}^{b}\left(\psi_{2}\left|y_{m}^{\prime}\right|^{2}+\psi_{1}\left|y_{m}\right|^{2}\right) d x \geq(1-r) \int_{a}^{b} \psi_{2}\left|y_{m}^{\prime}\right|^{2} d x \geq r_{0} \int_{a}^{b}\left|y_{m}^{\prime}\right|^{2} d x
$$

where $r_{0}=(1-r) \operatorname{Min}_{a \leq x \leq b} \psi_{2}(x)$. Thus, the sequence $\left\{\int_{a}^{b}\left|y_{m}^{\prime}\right|^{2} d x\right\}$ is 
bounded, and since each $y_{m}(x)$ vanishes at $a$ and $b$, the vector functions $y_{m}(x)$ are uniformly bounded on $a \leq x \leq b$. Moreover,

$$
\begin{aligned}
\int_{a}^{b}\left|z_{m}\right|^{2} d x & \leq \int_{a}^{b}\left|y_{m}^{\prime}+\left(z_{m}-y_{m}^{\prime}\right)\right|^{2} d x \\
& \leq 2 \int_{a}^{b}\left|y_{m}^{\prime}\right|^{2} d x+2 \int_{a}^{b}\left|z_{m}-y_{m}^{\prime}\right|^{2} d x \\
& \leq 2 \int_{a}^{b}\left|y_{m}^{\prime}\right|^{2} d x+2 M_{0}
\end{aligned}
$$

so that the sequence $\left\{\int_{a}^{b}\left|z_{m}\right|^{2} d x\right\}$ is bounded. Finally, with $f_{m}(x)$ continuing to denote $f\left(x, y_{m}(x), z_{m}(x)\right)$, we have

$$
\begin{aligned}
\int_{a}^{b}\left|z_{m}^{\prime}\right|^{2} d x & =\int_{a}^{b}\left|\left(z_{m}^{\prime}-f_{m}\right)+f_{m}\right|^{2} d x \\
& \leq 2 \int_{a}^{b}\left|z_{m}^{\prime}-f_{m}\right|^{2} d x+2 \int_{a}^{b}\left|f_{m}\right|^{2} d x \\
& \leq 2 M_{0}+2 \int_{a}^{b}\left|f_{m}\right|^{2} d x .
\end{aligned}
$$

As the vector functions $y_{m}(x),(m=1,2, \cdots)$, are bounded uniformly on $a \leq x \leq b$, in view of hypothesis $H_{3}$ and the result of Lemma 2.1, this latter inequality implies $\int_{a}^{b}\left|z_{m}^{\prime}\right|^{2} d x \leq K^{\prime \prime}+K^{\prime \prime} \int_{a}^{b}\left|z_{m}\right|^{2} d x+2 M_{0}$, for suitable constants $K^{\prime}, K^{\prime \prime}$. Hence, the two sequence $\left\{y_{m}(x)\right\},\left\{z_{m}(x)\right\}$ satisfy the hypotheses of Lemma 2.2, and we conclude that there exist subsequences, which will be denoted simply by $\left\{y_{m}(x)\right\}$ and $\left\{z_{m}(x)\right\}$, and a pair of functions $y(x), z(x)$ in $A_{2}$, such that $y_{m}(x) \rightarrow y(x)$ and $z_{m}(x) \rightarrow z(x)$ uniformly on $a \leq x \leq b$, while $y_{m}^{\prime}(x) \rightarrow y^{\prime}(x)$ and $z_{m}^{\prime}(x) \rightarrow z^{\prime}(x)$ weakly on the same interval.

With $f_{m}(x)$ as above and $f(x)=f(x, y(x), z(x))$ we have

$$
I\left(y_{m}, z_{m}\right)=I(y, z)+I_{1, m}+I_{2, m},
$$

where

$$
I_{1, m}=\int_{a}^{b}\left[\left|\left(z_{m}-z\right)-\left(y_{m}^{\prime}-y^{\prime}\right)\right|^{2}+\left|\left(f_{m}-f\right)-\left(z_{m}^{\prime}-z^{\prime}\right)\right|^{2}\right] d x
$$

and

$$
\begin{aligned}
I_{2, m}=2 \int_{a}^{b}\left\{( y ^ { \prime } - z ) ^ { * } \left[\left(y_{m}^{\prime}\right.\right.\right. & \left.\left.-y^{\prime}\right)-\left(z_{m}-z\right)\right] \\
& \left.+\left(z^{\prime}-f\right)^{*}\left[\left(z_{m}^{\prime}-z^{\prime}\right)-\left(f_{m}-f\right)\right]\right\} d x .
\end{aligned}
$$

Since $y_{m}(x) \rightarrow y(x), z_{m}(x) \rightarrow z(x)$ uniformly, we also have $f_{m}(x) \rightarrow f(x)$ uniformly on $a \leq x \leq b$. This, and the fact that $y_{m}^{\prime} \rightarrow y^{\prime}, z_{m}^{\prime} \rightarrow z^{\prime}$ weakly on the same interval, implies that $I_{2, m} \rightarrow 0$ as $m \rightarrow \infty$. As $I_{1, m} \geq 0$, it follows that 


$$
I_{0}=\lim _{m \rightarrow \infty} I\left(y_{m}, z_{m}\right) \geq I(y, z) .
$$

On the other hand, the definition of $I_{0}$ requires $I_{0} \leq I(y, z)$, so that $I_{0}=I(y, z)$; that is, $(y, z)$ renders $I(y, z)$ a minimum in the class of function pairs $K$.

It follows that for arbitrary $\gamma(x), \zeta(x)$ in $A_{2}$ with $\gamma(a)=0=\eta(b)$, and $\theta$ a real parameter, the functional $I(y+\theta \eta, z+\theta \zeta)$ has a minimum at $\theta=0$, and therefore $(d / d \theta) I(y+\theta \eta, z+\theta \zeta)=0$ for $\theta=0$; that is,

$$
\int_{a}^{b}\left[\left(y^{* \prime}-z^{*}\right)\left(\eta^{\prime}-\zeta\right)+\left(z^{* \prime}-f^{*}\right)\left(\zeta^{\prime}-f_{y} \eta-f_{z} \zeta\right)\right] d x=0,
$$

where the arguments of $f, f_{y}, f_{z}$ are $x, y(x), z(x)$.

In view of $H_{5}$, (see [4; pp. 213-214]), for an arbitrary continuous function $g(x), a \leq x \leq b$, there exists a solution $(\eta(x), \zeta(x))$ of the differential system

$$
\begin{aligned}
& \eta^{\prime}-\zeta=0, \\
& \zeta^{\prime}-f_{y}(x, y(x), z(x)) \eta-f_{z}(x, y(x), z(x)) \zeta=g(x), \quad a \leq x \leq b, \\
& \eta(a)=0=\eta(b) .
\end{aligned}
$$

Therefore, $\int_{a}^{b}\left[z^{* \prime}-f^{*}(x, y, z)\right] g(x) d x=0$ for arbitrary $g(x)$ continuous on $a \leq x \leq b$, and consequently $z^{\prime}(x)-f(x, y(x), z(x))=0$ a.e. on the same interval. Relation (3.5), with $\eta(x)$ chosen identically zero on $a \leq x \leq b$, then yields $\int_{a}^{b} \zeta^{*}\left(y^{\prime}-z\right) d x=0$ for arbitrary $\zeta$ in $A_{2}$, and hence $y^{\prime}(x)=z(x)$ a.e. on $a \leq x \leq b$. From the relations $z(x)=z(a)+\int_{a}^{x} f(s, y(s), z(s)) d s$, $y(x)=\int_{a}^{x} z(s) d s$, it then follows that $y(x)$ and $z(x)$ are of class $C^{\prime}$, and that $y^{\prime}(x)=z(x), z^{\prime}(x)=f(x, y(x), z(x))$ for $a \leq x \leq b$, so that $y(x)$ is of class $C^{\prime \prime}$ and satisfies (1.2).

4. Existence of a solution of (1.1). For the system

$$
\begin{aligned}
y^{\prime \prime} & =f\left(x, y, y^{\prime}\right), \quad a \leq x \leq b, \\
y(a) & =y_{1}, \quad y(b)=y_{2},
\end{aligned}
$$

let $F(x, y, z) \equiv f\left(x, y+\lambda(x), z+\lambda^{\prime}(x)\right)-\lambda^{\prime \prime}(x)$, where $\lambda(x)$ is any vector function of class $C^{\prime \prime}$ on $a \leq x \leq b$ satisfying $\lambda(a)=y_{1}, \lambda(b)=y_{2}$. Then (1.1) is equivalent, with $u=y-\lambda$, to

$$
\begin{aligned}
u^{\prime \prime} & =F\left(x, u, u^{\prime}\right), \quad a \leq x \leq b, \\
u(a) & =0=u(b) .
\end{aligned}
$$

This leads us to formulate the following hypothesis.

$H_{4}^{*}$. There exists $\lambda(x)$ of class $C^{\prime \prime}$ on $a \leq x \leq b$ with $\lambda(a)=y_{1}$, $\lambda(b)=y_{2}$, and such that for arbitrary $\rho>0$ there exist scalar functions 
$\psi_{1}(x)=\psi_{1}(x ; \rho)$, continuous on $a \leq x \leq b, \psi_{2}(x)=\psi_{2}(x ; \rho)$ of class $C^{\prime}$ on $a \leq x \leq b$ with $\psi_{2}(x)>0$, and a constant $N=N(\rho)$ such that:

(a) the scalar differential system $\left(\psi_{2}(x) w^{\prime}\right)^{\prime}-\psi_{1}(x) w=0$ is nonoscillatory on $a \leq x \leq b$;

(b) the integral inequality

$$
2 \int_{a}^{b} y^{*} f\left(x, y+\lambda, z+\lambda^{\prime}\right) d x \geq \int_{a}^{b}\left[\left(\psi_{2}-1\right)\left|y^{\prime}\right|^{2}+\psi_{1}|y|^{2}\right] d x-N
$$

holds for all $y(x), z(x)$ in $A_{2}$ satisfying $y(a)=0=y(b)$ and

$$
\int_{a}^{b}\left|y^{\prime}-z\right|^{2} d x<\rho
$$

THEOREM 4.1. Under hypotheses $H_{1}, H_{2}, H_{3}, H_{4}^{*}, H_{5}$, the system (1.1) has a solution.

Let $F(x, y, z)=f\left(x, y+\lambda(x), z+\lambda^{\prime}(x)\right)-\lambda^{\prime \prime}(x)$, where $\lambda(x)$ is the function described in $H_{4}^{*}$. Clearly, $F(x, y, z)$ satisfies $H_{1}, H_{2}, H_{3}$. Since $f$ satisfies $H_{4}^{*}$, we have

$$
2 \int_{a}^{b} y^{*} f\left(x, y+\lambda, z+\lambda^{\prime}\right) d x \geq \int_{a}^{b}\left[\left(\psi_{2}-1\right)\left|y^{\prime}\right|^{2}+\psi_{1}|y|^{2}\right] d x-N
$$

for arbitrary $y(x), z(x)$ satisfying $y(a)=0=y(b)$ and $\int_{a}^{b}\left|y^{\prime}-z\right|^{2} d x \leq \rho$. Hence, for such $y(x), z(x)$ we have

$$
\begin{aligned}
2 \int_{a}^{b} y^{*} F(x, y, z) d x \geq & \int_{a}^{b}\left[\left(\psi_{2}-1\right)\left|y^{\prime}\right|^{2}+\psi_{1}|y|^{2}\right] d x-N-2 \int_{a}^{b} y^{*} \lambda^{\prime \prime}(x) d x \\
\geq & \int_{a}^{b}\left[\left(\psi_{2}-1\right)\left|y^{\prime}\right|^{2}+\left(\psi_{1}-\varepsilon\right)|y|^{2}\right] d x \\
& -\left(N+\frac{1}{\varepsilon} \int_{a}^{b}\left|\lambda^{\prime \prime}\right|^{2} d x\right)
\end{aligned}
$$

for any $\varepsilon>0$. But by Lemma 2.4, $\varepsilon$ can be chosen so small that $\left(\psi_{2} w^{\prime}\right)^{\prime}-\left(\psi_{1}-\varepsilon\right) w=0$ is still non-oscillatory on $a \leq x \leq b$. Thus, $F(x, y, z)$ satisfies $H_{4}$. Finally, one easily verifies that if $f(x, y, z)$ satisfies $H_{5}$ then $F(x, y, z)$ satisfies $H_{5}$. Thus, whenever $f(x, y, z)$ satisfies the hypotheses of Theorem 4.1, the corresponding function $F(x, y, z)$ of (4.1) satisfies the hypotheses of Theorem 3.1, so that the result of Theorem 4.1 is a direct corollary of Theorem 3.1 .

5. A comment on altered hypotheses. We note here that hypothesis $H_{4}$ is implied by the more restrictive but simpler hypotheses $H_{4}^{\prime}$ and $H_{4}^{\prime \prime}$. $H_{4}^{\prime}$. There exists a constant $C$ such that $\left|y^{*}\left(f\left(x, y, z_{2}\right)-f\left(x, y, z_{1}\right)\right)\right| \leq C|y|\left|z_{2}-z_{1}\right|$, for $\left(x, y, z_{1}\right),\left(x, y, z_{2}\right)$ in $\Omega$.

$H_{4}^{\prime \prime}$. There exist scalar functions $\psi_{1}(x)$, continuous on $a \leq x \leq b$, 
and $\psi_{2}(x)>0$ of class $C^{\prime}$ on $a \leq x \leq b$, and $a$ constant $N$ such that:

(a) the scalar differential system $\left(\psi_{2}(x) w^{\prime}\right)^{\prime}-\psi_{1}(x) w=0$ is nonoscillatory on $a \leq x \leq b$;

(b) the integral inequality

$$
2 \int_{a}^{b} y^{*} f\left(x, y, y^{\prime}\right) d x \geq \int_{a}^{b}\left[\left(\psi_{2}-1\right)\left|y^{\prime}\right|^{2}+\psi_{1}|y|^{2}\right] d x-N
$$

holds for all $y(x)$ in $A_{2}$ satisfying $y(a)=0=y(b)$.

To see that $H_{4}$ is implied by $H_{4}^{\prime}$ and $H_{4}^{\prime \prime}$ (assuming, of course, $H_{1}$, $\left.H_{2}\right)$, for $y(x), z(x)$ in $A_{2}$ and $\varepsilon>0$ we write,

$$
\begin{aligned}
2 \int_{a}^{b} y^{*} f(x, y, z) d x & =2 \int_{a}^{b} y^{*} f\left(x, y, y^{\prime}\right) d x+2 \int_{a}^{b} y^{*}\left[f(x, y, z)-f\left(x, y, y^{\prime}\right)\right] d x \\
& \geq 2 \int_{a}^{b} y^{*} f\left(x, y, y^{\prime}\right) d x-2 C \int_{a}^{b}|y|\left|y^{\prime}-z\right| d x \\
& \geq 2 \int_{a}^{b} y^{*} f\left(x, y, y^{\prime}\right) d x-C \varepsilon \int_{a}^{b}|y|^{2} d x-(C / \varepsilon) \int_{a}^{b}\left|y^{\prime}-z\right|^{2} d x \\
& \geq \int_{a}^{b}\left[\left(\psi_{2}-1\right)\left|y^{\prime}\right|^{2}+\left(\psi_{1}-C \varepsilon\right)|y|^{2}\right] d x-[(C \rho) / \varepsilon+N]
\end{aligned}
$$

for all $y(x), z(x)$ in $A_{2}$ with $y(a)=0=y(b)$ and $\int_{a}^{b}\left|y^{\prime}-z\right|^{2} d x<\rho$. Since $\varepsilon$ can be chosen so small that $\left(\psi_{2} w^{\prime}\right)^{\prime}-\left(\psi_{1}-\varepsilon C\right) w=0$ is still non-oscillatory on $a \leq x \leq b$, we see that $H_{4}^{\prime}$ and $H_{4}^{\prime \prime}$ imply $H_{4}$.

It is to be noted that if the elements of $f_{z}(x, y, z)$ are bounded for $(x, y, z)$ in $\Omega$, then $f(x, y, z)$ satisfies both $H_{3}$ and $H_{4}^{\prime}$.

6. An example. Let $f(x, y, z)=g(x, y)\left(1+z^{2}\right)^{1 / 2}$, where $z$ is a scalar and $g(x, y)$ is a scalar function of the scalars $x$ and $y$ satisfying the conditions :

(a) $g(x, y)$ and $g_{y}(x, y)$ are continuous for $a \leq x \leq b,-\infty<y<\infty$;

(b) $g_{y}(x, y) \geq 0$ for $a \leq x \leq b,-\infty<y<\infty$;

(c) there exists a constant $A>0$ such that if $|y| \geq A$ then

$$
y g(x, y) \geq 0, \quad a \leq x \leq b .
$$

One may verify that $f(x, y, z)$ satisfies hypotheses $H_{1}, H_{2}, H_{3}, H_{4}^{*}$, and $H_{5}$.

\section{PART II}

1. Introduction. Sansone $[8 ; \mathrm{pp} .445-450]$ has proved the existence and uniqueness of a solution of the scalar differential system

$$
\begin{aligned}
& y^{\prime \prime}=\psi(x) \phi(x, y), \quad 0<x<\infty, \\
& y(0)=y_{0}, \quad y(+\infty)=0, \\
& y \in C^{\prime} \text { on } \quad 0 \leq x<\infty,
\end{aligned}
$$


under assumptions which are related to hypotheses $H_{1}-H_{6}$ (see $\S \S 2,7$ ) of this paper. The product $\psi(x) \phi(x, y)$ appears in (1.1) to facilitate stating the hypotheses in such a way as to include the Fermi-Thomas system (see [8; p. 445]),

$$
\begin{aligned}
y^{\prime \prime} & =x^{-1 / 2} y^{3 / 2}, \\
y(0) & =1, \quad \lim _{x \rightarrow \infty} y(x)=0 .
\end{aligned}
$$

In this paper we consider solutions of a vector differential system, for which we prove an existence and uniqueness theorem which includes the results of Sansone.

The proof given in [8] may be considered in two parts. In the first part the author proves, in effect, that under his hypotheses the system

$$
\begin{aligned}
& y^{\prime \prime}=\psi(x) \phi(x, y), \quad 0<x<\infty, \\
& y(0)=y_{0}, \quad y(x) \text { bounded on } 0 \leq x<\infty, \\
& y \in C^{\prime} \text { on } \quad 0 \leq x<\infty,
\end{aligned}
$$

has a unique solution. Essential to Sansone's proof of this result is the fact that his hypotheses guarantee a local uniqueness property for solutions of

$$
y^{\prime \prime}=\psi(x) \phi(x, y) ;
$$

that is, under his hypotheses, (1.4) has for $0 \leq x_{0}<\infty$ exactly one solution satisfying $y\left(x_{0}\right)=y_{0}, y^{\prime}\left(x_{0}\right)=y_{0}^{\prime}$. The hypotheses of the present paper, however, are not strong enough to imply such local uniqueness, as will be shown by an example in $\S 2$. In the second phase of his proof, Sansone appeals to hypotheses which are designed to guarantee that the bounded solutions of (1.3) actually satisfy (1.1). In this paper we make a similar step, but again our hypothesis is weaker than the corresponding ones in [8], as will be made clear in $\S 7$.

Sections 2-5 of this paper present an existence and uniqueness theorem for a solution of the vector generalization of Sansone's system mentioned above. This proof is primarily by variational methods, and the solutions are shown to be characterized by an extremal property. In $\S 6$ there is given a different characterization of these solutions, while $\$ 7$ contains several theorems relating to the asymptotic behavior of solutions. Finally, $\S 8$ is devoted to properties of solutions of (2.1) as functions of initial values.

2. Formulation of the problem. Let $g(x, y)$ be a real-valued scalar function of the scalar $x$ and the $n$-dimensional vector $y=\left(y_{j}\right)$. We will denote by $g_{y}(x, y)$ the vector $\left(g_{y_{j}}(x, y)\right)$, and consider the problem of solving the vector differential system 


$$
\begin{aligned}
y^{\prime \prime}(x) & =g_{y}(x, y(x)), \quad 0<x<\infty, \\
y(0) & =y_{0}, \quad y(x) \text { bounded on } 0 \leq x<\infty,
\end{aligned}
$$

where $y(x) \in C^{\prime}$ on $0 \leq x<\infty$ and $y(x) \in C^{\prime \prime}$ on $0<x<\infty$. We will suppose that $g(x, y)$ has the form $g(x, y)=\psi(x) G(x, y)$, where $\psi(x)$ and $G(x, y)$ are real-valued functions which satisfy the following hypotheses:

$H_{1} . \quad G(x, y)$ is continuous in $(x, y)$ on $\Omega: 0 \leq x<\infty,|y|<\infty$, and $G(x, 0) \equiv 0$ for $0 \leq x<\infty$.

$H_{2} . \quad G_{y}(x, y)$ exists and is continuous in $(x, y)$ on $\Omega$.

$H_{3}$. $y^{*} G_{y}(x, y) \geq 0$ for $(x, y)$ on $\Omega$.

$H_{4}$. $\quad \eta^{*}\left[G_{y}(x, y+\eta)-G_{y}(x, y)\right] \geq 0$ for $(x, y),(x, \eta)$ on $\Omega$.

$H_{5} . \psi(x)$ is continuous and positive for $x>0$ and integrable on any finite closed interval $0 \leq x \leq A$.

It is to be noted that $g(x, y)$ may satisfy $H_{1}-H_{5}$ without the equation $y^{\prime \prime}=g_{y}(x, y)$ having the local uniqueness property mentioned in $\S 1$. Indeed, if we take

$$
g(x, y)= \begin{cases}8 y^{3 / 2}, & y \geq 0 \\ 0, & y \leq 0\end{cases}
$$

so that

$$
g_{y}(x, y)= \begin{cases}12 y^{1 / 2}, & y \geq 0 \\ 0, & y \leq 0\end{cases}
$$

it is easily verified that $g(x, y)$ satisfies $H_{1}-H_{5}$, with $\psi(x) \equiv 1$. However, the function $y_{1}(x)=\left(x-x_{0}\right)^{4}, x_{0}>0$, satisfies the equation $y^{\prime \prime}(x)=g_{y}(x, y(x))$, as does the function $y_{2}(x) \equiv 0$. Since we have $y_{1}\left(x_{0}\right)=y_{2}\left(x_{0}\right), y_{1}^{\prime}\left(x_{0}\right)=y_{2}^{\prime}\left(x_{0}\right)$, it follows that the local uniqueness property does not obtain here.

A few consequences of the above hypotheses are worthy of comment. First, observe that $H_{2}$ and $H_{3}$ imply $G_{y}(x, 0) \equiv 0$ for $0 \leq x<\infty$. Also, since $G(x, 0) \equiv 0$ by $H_{1}$, and

$$
G(x, y)=\int_{0}^{1}\left[\frac{d}{d s} G(x, s y) d s\right]=\int_{0}^{1} y^{*} G_{y}(x, s y) d s=\int_{0}^{1} s^{-1}\left(s y^{*} G_{y}(x, s y)\right) d s,
$$

we have by $H_{3}$ that $G(x, y) \geq 0$ on $\Omega$. Moreover, if $y(x)$ is continuous on $0 \leq x \leq A, A>0$, and $y^{\prime \prime}(x)$ exists and satisfies $y^{\prime \prime}(x)=g_{y}(x, y(x))$ for $0 \leq x \leq A$, then $y \in C^{\prime}$ on $0 \leq x \leq A$. To see this we write

$$
y^{\prime}(x)=y^{\prime}(A)-\int_{x}^{A} y^{\prime \prime}(t) d t=y^{\prime}(A)-\int_{x}^{A} \psi(t) G_{y}(t, y(t)) d t, \quad 0<x \leq A .
$$

Hence, $\lim _{x \rightarrow 0} y^{\prime}(x)$ exists. This, with the fact that $y(x)$ is continuous for $0 \leq x \leq A$, implies $y^{\prime}(0)$ exists and that $\lim _{x \rightarrow 0} y^{\prime}(x)=y^{\prime}(0)$.

Next we note that if $G(x, y)$ satisfies $H_{1}$ and $H_{2}$, then $H_{4}$ is equivalent to the statement that $G(x, y)$ is convex in $y$; that is, 


$$
G\left(x, y_{2}\right)-G\left(x, y_{1}\right)-\left(y_{2}-y_{1}\right)^{*} G_{y}\left(x, y_{1}\right) \geq 0
$$

for arbitrary $\left(x, y_{1}\right),\left(x, y_{2}\right)$ in $\Omega$. Finally, we note that the condition $G(x, 0) \equiv 0$ of $H_{1}$ is no essential restriction, since if $G(x, y)$ satisfies $H_{1}-H_{5}$ with the exception of this condition, then the function $G_{1}(x, y) \equiv$ $G(x, y)-G(x, 0)$ satisfies $H_{1}-H_{5}$ and presents the same differential system (2.1).

3. Some properties of solutions. In addition to the system (2.1), we will consider also the system

$$
\begin{aligned}
y^{\prime \prime}(x) & =g_{y}(x, y(x)), \quad 0 \leq a \leq x \leq b, \\
y(a) & =y_{a}, \quad y(b)=y_{b},
\end{aligned}
$$

where $y$ is of class $C^{\prime \prime}$ on $a \leq x \leq b$, with the obvious modification in case $a=0$. For these systems we prove the following theorem.

THEOREM 3.1. Under hypotheses $H_{1}-H_{5}$, the systems (2.1) and (3.1) have at most one solution.

We will give the proof for (2.1); the proof for (3.1) is similar. If $y_{1}(x)$ and $y_{2}(x)$ are two solutions of $(2.1)$, let $\eta(x)=y_{1}(x)-y_{2}(x)$. By $H_{4}$ and $H_{5}$ we have for $0<x<\infty$,

$$
0 \leq \eta^{*}\left[g_{y}\left(x, y_{2}+\eta\right)-g_{y}\left(x, y_{2}\right)\right]=\eta^{*}\left[g_{y}\left(x, y_{1}\right)-g_{y}\left(x, y_{2}\right)\right]=\eta^{*} \eta^{\prime \prime},
$$

and hence,

$$
\int_{0}^{x} \eta^{*}(t) \eta^{\prime \prime}(t) d t \geq 0, \quad 0<x<\infty
$$

Consequently, upon integration by parts, we get

$$
\eta^{*}(x) \eta^{\prime}(x) \geq \int_{0}^{x}\left|\eta^{\prime}\right|^{2} d t \geq 0
$$

Since $\left(|\eta|^{2}\right)^{\prime}=2 \eta^{*} \eta^{\prime}$ and $\left(\left|\eta^{2}\right|\right)^{\prime \prime}=2\left|\eta^{\prime}\right|^{2}+2 \eta^{*} \eta^{\prime \prime}$, it then follows that

$$
\left(|\eta(x)|^{2}\right)^{\prime} \geq 0 \text {, and }\left(|\eta(x)|^{2}\right)^{\prime \prime} \geq 0, \quad 0 \leq x<\infty .
$$

Consequently, either $\eta(x) \equiv 0,0 \leq x<\infty$, or else $|\eta(x)| \rightarrow \infty$ as $x \rightarrow \infty$. Since the latter is impossible, (2.1) has at most one solution.

The following result will be of use later.

LEMMA 3.1. If $g(x, y)$ satisfies $H_{1}-H_{5}$, and $y(x)$ is a solution of $y^{\prime \prime}(x)=g_{y}(x, y(x))$ on $0<x<\infty$ with $\int_{0}^{\infty}\left|y^{\prime}\right|^{2} d x<\infty$, then $y(x)$ is bounded on $0 \leq x<\infty$.

If $y(x)$ satisfies $y^{\prime \prime}=g_{y}(x, y)$, then, since $\left(|y|^{2}\right)^{\prime \prime}=2\left|y^{\prime}\right|^{2}+2 y^{*} y^{\prime \prime}=$ $2\left|y^{\prime}\right|^{2}+2 y^{*} g_{y}(x, y) \geq 0$, we know that either there is an $x_{1}$ such that 
$|y| \equiv 0$ for $x \geq x_{1}$, or else there is an $x_{2}$ such that $|y| \neq 0$ for $x \geq x_{2}$. In the latter case we have

$$
|y||y|^{\prime}=y^{*} y^{\prime}, \quad x \geq x_{2},
$$

and

$$
|y|^{3}|y|^{\prime \prime}=|y|^{2}\left(y^{*} y^{\prime \prime}\right)+\left(|y|^{2}\left|y^{\prime}\right|^{2}-\left(y^{*} y^{\prime}\right)^{2}\right) \geq 0, \quad x \geq x_{2},
$$

since $y^{*} y^{\prime \prime}=y^{*} g_{y}(x, y) \geq 0$. Hence, either $|y|^{\prime} \leq 0$ for $x \geq x_{2}$, in which case $\lim _{x \rightarrow \infty}|y(x)| \leq\left|y\left(x_{2}\right)\right|$, or there is an $\alpha>0$ and an $x_{3} \geq x_{2}$ such that $|y|^{\prime} \geq \alpha>0$ for $x \geq x_{3}$. In this latter case, for $x \geq x_{3}$ we have $|y|\left|y^{\prime}\right| \geq y^{*} y^{\prime}=|y||y|^{\prime} \geq \alpha|y|$, so that $\left|y^{\prime}\right| \geq \alpha>0$ and consequently. $\int_{0}^{\infty}\left|y^{\prime}\right|^{2} d x=\infty$. Since this is the only case in which $y(x)$ would be unbounded, we conclude that if $y(x)$ is unbounded then $\int_{0}^{\infty}\left|y^{\prime}\right|^{2} d x=\infty$.

4. A preliminary existence theorem. In what follows $I(y ; a, b)$ will denote the functional

$$
I(y ; a, b)=\int_{a}^{b}\left[\left|y^{\prime}\right|^{2}+2 g(x, ; y)\right] d x, \quad y(x) \text { in } K(a, b),
$$

where $K(a, b)$ is the class of absolutely continuous vector functions $y(x)$ with $\left|y^{\prime}(x)\right|^{2}$ integrable on $a \leq x \leq b$, and satisfying $y(a)=y_{a}, y(b)=y_{0}$. We prove the following result.

THeOREM 4.1. If $g(x, y)$ satisfies hypotheses $H_{1}-H_{5}$, then for any $a, b$ satisfying $0 \leq a<b$, the system (3.1) has a unique solution. Moreover, this solution is a unique minimizing function for $I(y ; a, b)$ in the class $K(a, b)$.

By $H_{5}$ and the fact that $g(x, y) \geq 0$, we see that $I(y ; a, b) \geq 0$ for $y$ in $K(a, b)$. Let $\bar{I}(a, b)$ denote the infimum of $I(y ; a, b)$ for $y$ in $K(a, b)$, and let $\left\{y_{m}(x)\right\}$ be a sequence of elements of $K(a, b)$ such the $I\left(y_{m} ; a, b\right) \rightarrow$ $\vec{I}(a, b)$. As $g\left(x, y_{m}(x)\right) \geq 0$ on $a<x \leq b$, we have

$$
\int_{a}^{b}\left|y_{m}^{\prime}\right|^{2} d x=I\left(y_{m} ; a, b\right)-2 \int_{a}^{b} g\left(x, y_{m}\right) d x \leq I\left(y_{m} ; a, b\right),
$$

so that there exists an $N$ such that $\int_{a}^{b}\left|y_{m}^{\prime}\right|^{2} d x<N$ for $m=1,2, \cdots$. Moreover, for $a \leq x \leq b$,

$$
\left|y_{m}(x)-y_{a}\right|^{2}=\left|\int_{a}^{x} y_{m}^{\prime}(t) d t\right|^{2} \leq(x-a) \int_{0}^{x}\left|y_{m}^{\prime}\right|^{2} d t \leq(b-a) \int_{a}^{b}\left|y_{m}^{\prime}\right|^{2} d t,
$$

so that $\left|y_{m}(x)-y_{a}\right| \leq[(b-a) N]^{1 / 2}$, and hence $\left|y_{m}(x)\right| \leq\left|y_{a}\right|+[(b-a) N]^{1 / 2}$. Consequently, we may use Lemma 2.2 of Part I to conclude that there is a subsequence, which we will denote again by $\left\{y_{m}(x)\right\}$, and a function 
$y(x)$ in $K(a, b)$, such that $y_{m}(x) \rightarrow y(x)$ uniformly on $a \leq x \leq b$, and $y_{m}^{\prime}(x) \rightarrow y^{\prime}(x)$ weakly on this interval.

From the identity

$$
\begin{aligned}
I\left(y_{m} ; a, b\right)-I(y ; a, b)=\int_{a}^{b}\left[\left|y_{m}^{\prime}-y^{\prime}\right|^{2}\right. & +2\left(g\left(x, y_{m}\right)-g(x, y)\right) \\
& \left.+2\left(y_{m}^{\prime}-y^{\prime}\right)^{*} y^{\prime}\right] d x,
\end{aligned}
$$

and the fact that $y_{m}(x) \rightarrow y(x)$ uniformly on $a \leq x \leq b$ while $y_{m}^{\prime}(x) \rightarrow y^{\prime}(x)$ weakly on this interval, one obtains the lower semi-continuity relation

$$
\bar{I}(a, b)=\lim _{m \rightarrow \infty} I\left(y_{m} ; a, b\right) \geq I(y ; a, b) .
$$

Since the definition of $\bar{I}(a, b)$ requires that $\bar{I}(a, b) \leq I(y ; a, b)$, we see that $\bar{I}(a, b)=I(y ; a, b)$; that is, $y(x)$ minimizes $I(y ; a, b)$ in the class $K(a, b)$.

It follows that if $\eta(x)$ is absolutely continuous with $\eta(a)=0=\eta(b)$ and $\left|\eta^{\prime}(x)\right|^{2}$ is integrable on $a \leq x \leq b$, and $\theta$ is a real parameter, then $I(y+\theta \eta ; a, b)$ has a minimum at $\theta=0$. From this it follows that $(d / d \theta) I(y+\theta \eta ; a, b)=0$ at $\theta=0$; that is,

$$
\int_{a}^{b}\left[\eta^{\prime *} y^{\prime}+\eta^{*} g_{y}(x, y)\right] d x=0
$$

In particular, this last equality holds for arbitrary $\eta$ of class $C^{\prime \prime}$ on a $\leq x \leq b$ with $\eta(a)=0=\eta(b)=\eta^{\prime}(a)=\eta^{\prime}(b)$, and for such an $\eta$ integration by parts leads to

$$
\int_{a}^{b} \eta^{\prime \prime *}\left[y(x)-\int_{a}^{x} d s \int_{a}^{s} g_{y}(t, y(t)) d t\right] d x=0 .
$$

By the fundamental lemma of the calculus of variations, there exist constant vectors $\xi_{1}$ and $\xi_{2}$ such that

$$
y(x)=\int_{a}^{x} d s \int_{a}^{s} g_{y}(t, y(t)) d t+\xi_{1} x+\xi_{2}, \quad a \leq x \leq b .
$$

Therefore, $y^{\prime \prime}(x)$ exists and satisfies

$$
y^{\prime \prime}(x)=g_{y}(x, y(x)), \quad a \leq x \leq b,
$$

with the understanding that if $a=0$, then $y^{\prime \prime}(x)$ may fail to exist at $x=0$. Since $y(a)=y_{a}, y(b)=y_{b}$, it follows that $y(x)$ satisfies (3.1). The uniqueness of this solution follows from Theorem 3.1. Moreover, since the above argument shows that any function of class $K(a, b)$ that minimizes $I(y ; a, b)$ is a solution of (3.1), it follows that the above determined $y(x)$ is the unique minimizing function for $I(y ; a, b)$ in $K(a, b)$. 
5. An existence theorem for a solution of (2.1). In what follows, $K$ will denote the class of absolutely continuous vector functions $y(x)$ with $\left|y^{\prime}\right|^{2}$ integrable on $0 \leq x<\infty$ and satisfying $y(0)=y_{0}, I(y ; 0, \infty)<\infty$, where

$$
I(y ; 0, \infty)=\int_{0}^{\infty}\left[\left|y^{\prime}\right|^{2}+2 g(x, y)\right] d x .
$$

We now prove the following result. .

THEOREM 5.1. Under hypotheses $H_{1}-H_{5}$ the system (2.1) has a unique solution; moreover, this solution is a unique minimizing function for $I(y ; 0, \infty)$ in the class $K$.

Let $\left\{y_{m}(x)\right\}, m=1,2, \cdots$, be a sequence of functions in $K$ such that $I\left(y_{m} ; 0, \infty\right) \rightarrow \bar{I}$, where $\bar{I}$ denotes the infimum of $I(y ; 0, \infty)$ for $y$ in $K$. Then the non-negativeness of $g(x, y)$ implies that the sequence $\left\{\int_{0}^{\infty}\left|y_{m}^{\prime}\right|^{2} d x\right\}$ is bounded, and since $y_{m}(0)=y_{0}$ for every $m$, as in the proof of Theorem 4.1, the $y_{m}(x)$ are uniformly bounded on each finite interval. Hence, by Lemma 2.2 of Part I, there is a subsequence, say $\left\{y_{m}(x)\right\}$ again, and an absolutely continuous function $y(x)$, such that on each finite interval $y_{m}(x) \rightarrow y(x)$ uniformly, and $y_{m}^{\prime}(x) \rightarrow y^{\prime}(x)$ weakly. Now for any $A>0$ we have $I\left(y_{m} ; 0, \infty\right) \geq I\left(y_{m} ; 0, A\right)$; moreover, as in $\S 4$ we have

$$
I\left(y_{m} ; 0, A\right)-I(y ; 0, A) \geq 2 \int_{0}^{A}\left[\left(g\left(x, y_{m}\right)-g(x, y)\right)+\left(y_{m}^{\prime}-y^{\prime}\right)^{*} y^{\prime}\right] d x
$$

and consequently $\lim \inf _{m \rightarrow \infty} I\left(y_{m} ; 0, A\right) \geq I(y ; 0, A)$. Hence

$$
\bar{I}=\lim _{m \rightarrow \infty} I\left(y_{m} ; 0, \infty\right) \geq I(y ; 0, A), \quad A>0,
$$

and finally,

$$
\bar{I} \geq I(y ; 0, \infty)=\lim _{A \rightarrow \infty} I(y ; 0, A) .
$$

In particular, this latter relation implies that $y(x)$ is in $K$, and in view of the definition of $\bar{I}$ we have $I(y ; 0, \infty) \geq \bar{I}$, so that $I(y ; 0, \infty)=\bar{I}$. That is, $y(x)$ minimizes $I(y ; 0, \infty)$ in the class $K$.

Now on any finite interval $0 \leq x \leq A$, the thus determined $y(x)$ must coincide with the unique vector function which minimizes $I(y ; 0, A)$ in the class $K(0, A)$ of curves joining $\left(0, y_{0}\right)$ and $(A, y(A))$, for otherwise one could piece together a curve which would give $I(y ; 0, \infty)$ a smaller value than does $y(x)$. By Theorem 4.1 it then follows that $y(x)$ satisfies $y^{\prime \prime}(x)=g_{y}(x, y(x))$ on $0<x \leq A$, where $A$ is arbitrary, and consequently $y^{\prime \prime}(x)=g_{y}(x, y(x))$ on $0<x<\infty$. Since $I(y ; 0, \infty)$ is finite, Lemma 3.1 implies that $y(x)$ is bounded on $0 \leq x<\infty$ and therefore is a solution of (2.1). The uniqueness of this solution follows from Theorem 3.1, 
Inasmuch as we have actually shown that any $y(x)$ that minimizes $I(y ; 0, \infty)$ in $K$ is a solution of $(2.1)$, the uniqueness of $y(x)$ as a minimizing function follows from its uniqueness as a solution of (2.1).

\section{A further characterization of solutions of (2.1).}

THEOREM 6.1. Suppose that hypotheses $H_{1}-H_{5}$ are satisfied, and $y_{\infty}(x)$ is the unique solution of (2.1) guaranteed by Theorem 5.1. If, for a given vector, $\xi, y=y_{N}(x, \xi), 0 \leq x \leq N$, is the solution of

$$
\begin{gathered}
y^{\prime \prime}=g_{y}(x, y(x)), \\
y(0)=y_{0}, \quad y(N)=\xi, \quad N=1,2, \cdots,
\end{gathered}
$$

then $y_{N}(x, \xi) \rightarrow y_{\infty}(x)$ and $y_{N}^{\prime}(x, \xi) \rightarrow y_{\infty}^{\prime}(x)$ uniformly on each subinterval $0 \leq x \leq A$.

We will suppose in what follows that the definition of $y_{N}(x, \xi)$ has been extended so that $y_{N}(x, \xi)=\xi$ for $x \geq N$. The inequality $\left(\left|y_{N}(x, \xi)\right|^{2}\right)^{\prime \prime} \geq 0$ and the end conditions (6.1b) then imply that

$$
\left|y_{N}(x, \xi)\right| \leq \operatorname{Max}\left(\left|y_{0}\right|,|\xi|\right), \quad 0 \leq x<\infty, \quad N=1,2, \cdots .
$$

Moreover, the identity

$$
\begin{gathered}
y_{N}^{\prime}(x, \xi)=\frac{1}{A}\left[y_{N}(A, \xi)-y_{0}-\int_{0}^{A} d s \int_{x}^{s} g_{y}\left(t, y_{N}(t, \xi)\right) d t\right], \\
0 \leq x \leq A, \quad N>A,
\end{gathered}
$$

shows that the sequence $\left\{\left|y_{N}^{\prime}(x, \xi)\right|\right\}$ is uniformly bounded on $0 \leq x \leq A$. Consequently, the sequence $\left\{y_{N}(x, \xi)\right\}$ is uniformly bounded and equicontinuous on any finite interval, so that we may select a subsequence $\left\{y_{N_{j}}(x, \xi)\right\}$ which converges uniformly on any finite interval to a continuous function $y(x)$. From (4.1) it follows that if $\eta(x)$ is of class $C^{\prime \prime}$ on $0 \leq x<\infty$, and $\eta(0)=0=\eta^{\prime}(0)=\eta^{\prime}(A), \eta(x) \equiv 0$ for $x \geq A$, then

$$
\int_{0}^{A} \eta^{\prime \prime *} *\left[y_{N_{j}}(x, \xi)-\int_{0}^{x} d s \int_{0}^{s} g_{y}\left(t, y_{N_{j}}(t, \xi)\right) d t\right] d x=0, \quad N>A .
$$

Since $y_{N_{j}}(x, \xi) \rightarrow y(x)$ uniformly on $0 \leq x \leq A$, we then have

$$
\int_{0}^{A} \eta^{\prime \prime *}\left[y(x)-\int_{0}^{x} d s \int_{0}^{s} g_{y}(t, y(t)) d t\right] d x=0 .
$$

As before, application of the fundamental lemma of the calculus of variation yields the result that $y^{\prime \prime}(x)$ exists and $y^{\prime \prime}=g_{y}(x, y)$ for $0<x \leq A$. Since $A$ is arbitrary, it follows that $y^{\prime \prime}(x)=g_{y}(x, y(x))$ on $0<x<\infty$. Moreover, $y(0)=y_{0}$, while the relation (6.2) shows that $y(x)$ is bounded on $0 \leq x<\infty$, so that in view of Theorem 5.1 we have $y(x)=y_{\infty}(x)$.

Now for $0 \leq x_{0}<\infty$, let $\eta$ be any accumulation point of the bounded 
sequence $\left\{y_{N}\left(x_{0}, \xi\right)\right\}$, and let a subsequence $\left\{y_{N_{i}}\left(x_{0}, \xi\right)\right\}$ be chosen such that $y_{N_{i}}\left(x_{0}, \xi\right) \rightarrow \eta$. Then, as before, the sequence $\left\{y_{N_{i}}(x, \xi)\right\}$ is uniformly bounded and equicontinuous on any finite interval, so that we may select a subsequence which approaches $y_{\infty}(x)$ on $0 \leq x<\infty$. Consequently, the sequence $\left\{Y_{N}\left(x_{0}, \xi\right)\right\}$ has only one accumulation point, namely $\eta=y_{\infty}\left(x_{0}\right)$, from which it follows that $y_{N}(x, \xi) \rightarrow y_{\infty}(x)$ for $0 \leq x<\infty$.

With $\zeta_{N}(x)=y_{N}(x, \xi)-y_{\infty}(x)$, as in the proof of Theorem 3.1 we have that $\left(\left|\zeta_{N}(x)\right|^{2}\right)^{\prime} \geq 0,0 \leq x \leq N$. This implies that for any $A>0$ and $N>A$ we have $\left|\zeta_{N}(x)\right| \leq\left|\zeta_{N}(A)\right|$ on $0 \leq x \leq A$, and thus $y_{N}(x, \xi) \rightarrow$ $y_{\infty}(x)$ uniformly on $0 \leq x \leq A$.

The fact that $y_{N}^{\prime}(x, \xi) \rightarrow y_{\infty}^{\prime}(x)$ uniformly on $0 \leq x \leq A$ now follows from (6.3), and the corresponding identity obtained by replacing $y_{N}(x, \xi)$ by $y_{\infty}(x)$.

7. Asymptotic behavior of solutions of (2.1). At this point we introduce the following hypotheses:

$H_{6}$. For each $c>0$ there is an $x_{c} \geq 0$ and a $\Psi(x, c) \geq 0$ with $x \Psi(x, c)$ integrable on every finite subinterval of $x_{c} \leq x<\infty, \int_{x_{c}}^{\infty} x \Psi(x, c) d x=\infty$, and such that for $x \geq x_{c},|y| \geq c$ we have $y^{*} g_{y}(x, y) \geq \Psi(x, c)$.

$H_{7}$. If $y(x)$ is in $C^{\prime}$ and $|y(x)| \geq c>0$ for $0 \leq x<\infty$, then $I(y(x) ; 0, \infty)=\infty$.

We have the following result.

THEOREM 7.1. If in addition to $H_{1}-H_{5}$, either $H_{6}$ or $H_{7}$ is also satisfied, then any solution of (2.1) approaches zero as $x \rightarrow \infty$.

If $y(x)$ is a solution of (2.1), then $\left(|y|^{2}\right)^{\prime \prime}=2 y^{*} y^{\prime \prime}+2\left|y^{\prime}\right|^{2} \geq 0$. Since $y(x)$ is bounded on $0 \leq x<\infty$, it follows that $\left(|y|^{2}\right)^{\prime} \leq 0$, so that either $|y(x)|$ is bounded away from zero or else $y(x) \rightarrow 0$. If $H_{7}$ is satisfied then, in view of the fact that $I(y(x) ; 0, \infty)$ is finite for $y(x)$ a solution of (2.1), it follows that $|y(x)|$ cannot be bounded from zero; that is, $y(x) \rightarrow 0$.

Suppose now that $H_{6}$ is satisfied. As was noted in the preceding paragraph, $\left(|y|^{2}\right)^{\prime}$ is non-decreasing and non-positive, so that $\lim _{x \rightarrow \infty}\left(|y|^{2}\right)^{\prime}$ exists. This limit is zero, since $|y|^{2}$ is non-negative, and hence $\lim _{x \rightarrow \infty} y^{*} y^{\prime}=0$. This fact leads to the following relations,

$$
\begin{aligned}
& -2 y^{*}(x) y^{\prime}(x)=\int_{x}^{\infty}\left(|y|^{2}\right)^{\prime \prime} d t=2 \int_{x}^{\infty}\left(y^{*} y^{\prime \prime}+\left|y^{\prime}\right|^{2}\right) d t, \\
& -2 y^{*}(x) y^{\prime}(x)=2 \int_{x}^{\infty}\left(y^{*}(t) g_{y}(t, y(t))+\left|y^{\prime}(t)\right|^{2}\right) d t \\
& -y^{*}(x) y^{\prime}(x) \geq \int_{x}^{\infty} y^{*}(t) g_{y}(t, y(t)) d t
\end{aligned}
$$

Integration now yields 


$$
\frac{1}{2}|y(x)|^{2}-\frac{1}{2}|y(A)|^{2} \geq \int_{x}^{A} d s \int_{s}^{\infty} y^{*} g_{y} d t
$$

and hence

$$
\frac{1}{2}|y(x)|^{2} \geq \int_{x}^{A} d s \int_{s}^{\infty} y^{*} g_{y} d t .
$$

Finally, upon integration by parts we obtain

$$
\frac{1}{2}|y(x)|^{2} \geq A \int_{A}^{\infty} y^{*} g_{y} d t-x \int_{x}^{\infty} y^{*} g_{y} d t+\int_{x}^{A} s y^{*}(s) g_{y}(s, y(s)) d s .
$$

If there is a $c>0$ such that $|y(x)| \geq c$ on $x_{c} \leq x<\infty$, then by $H_{6}$ it follows that for all $x$ and $A$ satisfying $x_{c} \leq x<A<\infty$

$$
\frac{1}{2}|y(x)|^{2} \geq \int_{x}^{A} s \Psi(s, c) d s-x \int_{x}^{\infty} y^{*} g_{y}(t, y(t)) d t .
$$

But this implies that $\int_{x}^{\infty} s \Psi(s, c) d s<\infty$, contrary to assumption. Thus, there is no $c>0$ such that $|y(x)| \geq c$ on an interval of the form $x_{c} \leq$ $x<\infty$, and since $|y(x)|$ is non-increasing it follows that $|y(x)| \rightarrow 0$ as $x \rightarrow \infty$.

In connection with the comments in $\S 1$ of this paper, it is to be noted that the hypotheses used in [8] to establish the analogue of our Theorem 7.1 correspond to the assumption that the $\Psi(x)$ of $H_{6}$ satisfies $\int_{0}^{\infty} \Psi(x) d x=\infty$, instead of the weaker requirement made here.

For the next two theorems we will make use of the following hypothesis.

$H_{8}$. There exists a function $\phi(x)$ such that

$$
\begin{aligned}
& \left|g_{y}\left(x, y_{1}\right)-g_{y}\left(x, y_{2}\right)\right| \leq \phi(x)\left|y_{2}-y_{1}\right| \\
& \text { for } 0 \leq x<\infty,\left|y_{1}\right|<\infty,\left|y_{2}\right|<\infty,
\end{aligned}
$$

where $\phi(x) \geq 0, x \phi(x)$ is integrable on any finite subinterval of $0 \leq$ $x<\infty$, and $\int_{0}^{\infty} x \phi(x) d x<\infty$.

We prove the following theorem :

TheOREM 7.2. If $g(x, y)$ satisfies $H_{1}, H_{2}, H_{5}, H_{8}$, and $g_{y}(x, 0) \equiv 0$, and if $\alpha$ is any constant vector, then there is a unique solution $y(x)$ of $y^{\prime \prime}=g_{y}(x, y)$ for which $y(x) \rightarrow \alpha$ as $x \rightarrow \infty$.

Let $G(x)=\int_{x}^{\infty} t \phi(t) d t$. Imitating Hille [3; p. 238], we consider the following successive approximations corresponding to a given vector $\alpha$,

$$
\begin{gathered}
y_{0}(x) \equiv \alpha, \quad 0 \leq x<\infty . \\
y_{k}(x)=\alpha+\int_{x}^{\infty}(t-x) g_{y}\left(t, y_{k-1}(t)\right) d t, \quad 0 \leq x<\infty .
\end{gathered}
$$


We will show by induction that for $0 \leq x<\infty$,

(a) $y_{k}(x)$ is defined;

(b) $\left|y_{k}(x)-y_{k-1}(x)\right| \leq|\alpha| \frac{[G(x)]^{k}}{k !} \leq \frac{|\alpha|[G(0)]^{k}}{k !}, \quad k=1,2, \cdots$

We have $\left|y_{1}(x)-y_{0}(x)\right|=\left|\int_{0}^{\infty}(t-x) g_{y}(t, \alpha) d t\right|$. The integral here exists since on $x \leq t<\infty$ we have $|t-x|\left|g_{y}(t, \alpha)\right| \leq t\left|g_{y}(t, \alpha)\right| \leq t \phi(t)|\alpha|$, which is integrable on $x \leq t<\infty$. Moreover,

$$
\left|y_{1}(x)-y_{0}(x)\right| \leq|\alpha| \int_{x}^{\infty} t \phi(t) d t=|\alpha| G(x),
$$

so that (7.1) is satisfied for $k=1$.

Suppose (7.1) is true for $k=1,2, \cdots, N-1$. Then $y_{N}(x)$ is defined, since $\mid g_{y}\left(t, y_{N-1}(t)|\leq \phi(t)| y_{N-1}(t) \mid\right.$, where $y_{N-1}(t)$ is bounded on $0 \leq t<\infty$. Moreover,

$$
\begin{aligned}
\left|y_{N}(x)-y_{N-1}(x)\right| & =\left|\int_{x}^{\infty}(t-x)\left(g_{y}\left(t, y_{N-1}(t)\right)-g_{y}\left(t, y_{N-2}(t)\right)\right) d t\right| \\
& \leq \int_{k}^{\infty} t \phi(t)\left|y_{N-1}(t)-y_{N-2}(t)\right| d t \\
& \leq \frac{|\alpha|}{(N-1) !} \int_{x}^{\infty} t \phi(t) G^{N-1}(t) d t .
\end{aligned}
$$

Since $G^{N-1}(t)$ is bounded, all the integrals above exist. Hence,

$$
\left|y_{N}(x)-y_{N-1}(x)\right| \leq \frac{-|\alpha|}{(N-1) !} \int_{x}^{\infty}[G(t)]^{N-1} G^{\prime}(t) d t=\frac{|\alpha|[G(x)]^{N}}{N !}
$$

Now $y_{N}(x)-\alpha=\left(y_{1}-y_{0}\right)+\left(y_{2}-y_{1}\right)+\cdots+\left(y_{N}-y_{N-1}\right)$, and the series $\sum_{k=1}^{\infty}\left|y_{k}(x)-y_{k-1}(x)\right|$ converges uniformly on $0 \leq x<\infty$ by (7.1b). Hence $y(x)=\lim _{N \rightarrow \infty} y_{N}(x)$ exists ; moreover $y(x)$ is continuous on $0 \leq x<\infty$ and satisfies $|y(x)| \leq|\alpha| \exp \{G(x)\}$. Therefore $|y(x)|$ is bounded on $0 \leq x<\infty$, and $H_{8}$ with the uniform convergence of $\left\{y_{N}(x)\right\}$ on $0 \leq x<\infty$ implies $y(x)=\alpha+\int_{x}^{\infty}(t-x) g_{y}(t, y(t)) d t$, so that

$$
\begin{gathered}
y^{\prime \prime}(x)=g_{y}(x, y(x)), \quad 0<x<\infty, \\
\lim _{x \rightarrow \infty} y(x)=\alpha .
\end{gathered}
$$

If $Y(x)$ satisfies $Y^{\prime \prime}(x)=g_{y}(x, Y(x))$ on $0<x<\infty$ and $Y(x) \rightarrow \beta$ as $x \rightarrow \infty$, then the integral $\int_{x}^{\infty}(t-x) g_{y}(t, Y(t)) d t, 0 \leq x<\infty$, exists and $\gamma(x) \equiv Y(x)-\beta-\int_{x}^{\infty}(t-x) g_{y}(t, Y(t)) d t$ is such that $\eta^{\prime \prime}(x)=0$, $0<x<\infty$, and $\gamma(x) \rightarrow 0$ as $x \rightarrow \infty$. Hence, $\gamma(x) \equiv 0$ and $Y(x)=\beta+$ $\int_{x}^{\infty}(t-x) g_{y}(t, Y(t)) d t$. With $y(x)$ as above we then have 


$$
\begin{aligned}
|y(x)-Y(x)| & =\left|\alpha-\beta+\int_{x}^{\infty}(t-x)\left[g_{y}(t, y(t))-g_{y}(t, Y(t))\right] d t\right| \\
& \leq|\alpha-\beta|+\int_{x}^{\infty} t \phi(t)|y(t)-Y(t)| d t
\end{aligned}
$$

so that by a simple modification of Gronwall's lemma, (see [1; p. 35]), it follows that

$$
|y(x)-Y(x)| \leq|\alpha-\beta| \exp \{G(x)\} .
$$

If $\beta=\alpha$ then $Y(x) \equiv y(x)$, which proves the uniqueness of solutions of $y^{\prime \prime}=g_{y}(x, y)$ with given limit as $x \rightarrow \infty$. Moreover, $|y(x)-Y(x)| \leq$ $|\alpha-\beta| \exp \{G(0)\}$, so that we have the following corollary.

CoRollary 7.1. The solution $y(x)$ described in Theorem 7.2 is a continuous function of $\alpha=y(\infty)$.

We now prove the following theorem on the order of growth of solutions.

THEOREM 7.3. If $g(x, y)$ satisfies $H_{1}, H_{2}, H_{5}, H_{8}$, and $g_{y}(x, 0) \equiv 0$, and if $y(x)$ satisfies $y^{\prime \prime}=g_{y}(x, y)$ on $0<x<\infty$, then $\eta=\lim _{x \rightarrow \infty} y^{\prime}(x)$ exists and is finite, and $y(x)=x[\eta+o(1)]$.

Note the $H_{8}$ implies $\left|g_{y}(x, y)\right| \leq \phi(x)|y|$, which is all that is needed here. If $y(x)$ satisfies $y^{\prime \prime}=g_{y}(x, y)$, then following Bellman [1; p. 114] we write

$$
y(x)=y(0)+x y^{\prime}(0)+\int_{0}^{x}(x-t) g_{y}(t, y(t)) d t .
$$

Hence, for $x \geq 1$,

$$
|y(x)| \leq x\left(|y(0)|+\left|y^{\prime}(0)\right|\right)+x \int_{0}^{x} \phi(t)|y(t)| d t
$$

or

$$
\frac{|y(x)|}{x} \leq\left(|y(0)|+\left|y^{\prime}(0)\right|\right)+\int_{0}^{x} t \phi(t) \frac{|y(t)|}{t} d t .
$$

Therefore, by Gronwall's lemma, (see [1; p. 35]),

$$
\frac{|y(x)|}{x} \leq\left(|y(0)|+\left|y^{\prime}(0)\right|\right) \exp \left\{\int_{0}^{x} t \phi(t) d t\right\},
$$

and hence there is a constant $M$ such that

$$
|y(x)| \leq M x, \quad x \geq 1 .
$$

Now for $x \geq 1$ we have 


$$
\int_{1}^{x}\left|g_{y}(t, y(t))\right| d t \leq \int_{1}^{x} \phi(t)|y(t)| d t \leq M \int_{1}^{x} t \phi(t) d t,
$$

so that $\int_{0}^{\infty}\left|g_{y}(t, y(t))\right| d t$ exists. Since

$$
y^{\prime}(x)=y^{\prime}(0)+\int_{0}^{x} g_{y}(t, y(t)) d t,
$$

we have that $y^{\prime}(x) \rightarrow \eta$ as $x \rightarrow \infty$, where

$$
\eta=y^{\prime}(0)+\int_{0}^{\infty} g_{y}(t, y(t)) d t
$$

The final equality in the theorem is a ready consequence of this finite limit of $y^{\prime}(x)$.

8. Behavior of solutions of (2.1) with respect to initial values. We continue to suppose here that $H_{1}-H_{5}$ are satisfied, but not necessarily any other hypotheses. Let $y_{1}(x), y_{2}(x)$ be two bounded solutions of $y^{\prime \prime}=$ $g_{y}(x, y)$ on $0 \leq x<\infty$, and set $\eta(x)=y_{1}(x)-y_{2}(x)$. Then by $H_{4}$, we have $\eta^{*} \eta^{\prime \prime} \geq 0$, so that $\left(|\eta|^{2}\right)^{\prime \prime}=2\left|\eta^{\prime}\right|^{2}+2 \eta^{*} \eta^{\prime \prime} \geq 0$. Since $\eta(x)$ is bounded, we must have $|\eta(x)|$ non-increasing; in particular, $|\eta(x)| \leq|\eta(0)|$ on $0 \leq x<\infty$. Suppose now we denote by $y(x ; \alpha)$ the unique bounded solution of $y^{\prime \prime}=g_{y}(x, y)$ which satisfies $y(0 ; \alpha)=\alpha$. Then $y(x ; \alpha)$ is continuous in $x$ and $\alpha$ jointly, as may be seen from the inequality

$$
\begin{aligned}
|y(\bar{x} ; \bar{\alpha})-y(x, \alpha)| & \leq|y(\bar{x} ; \bar{\alpha})-y(\bar{x} ; \alpha)|+|y(\bar{x} ; \alpha)-y(x ; \alpha)|, \\
& \leq|\bar{\alpha}-\alpha|+|y(\bar{x} ; \alpha)-y(x ; \alpha)| .
\end{aligned}
$$

Moreover, for any $A>0$,

$$
y^{\prime}(x ; \alpha)=\frac{1}{A}\left[y(A ; \alpha)-y(0 ; \alpha)-\int_{0}^{A} d s \int_{x}^{s} g_{y}(t, y(t ; \alpha)) d t\right],
$$

so that $y^{\prime}(x ; \alpha)$ is also continuous in $x$ and $\alpha$.

We turn now to the question of differentiability of solutions with respect to initial values. The derivation of our results will involve the use of a lemma, the proof of which is based on certain theorems due to W. T. Reid. In [6], Reid has discussed a class of non-oscillatory linear matrix differential equations which includes as a special case the matrix equation

$$
U^{\prime \prime}=P(x) U, \quad a \leq x<\infty
$$

where $P(x)$ is a non-negative definite symmetric matrix with continuous real-valued elements. As shown in Theorem 6.1 of [6], if $U(x)$ is a solution of (8.2) which is non-singular on a subinterval $b \leq x<\infty$, and the necessarily constant matrix $U^{*}(x) U^{\prime}(x)-U^{* \prime}(x) U(x)$ is the zero matrix, then 


$$
M(b ; U)=\lim _{x \rightarrow \infty}\left(\int_{b}^{x} U^{-1}(t) U^{*-1}(t) d t\right)^{-1}
$$

exists and is finite. Moreover, such a $U(x)$ is a principal solution of (8.2) in the sense of Reid [6] if and only if $M(b ; U)=0$. In addition, a principal solution $U(x)$ is characterized by $U(x)=U_{b, \infty}(x) C$, where $C$ is a non-singular constant matrix and $U_{b, \infty}=\lim _{t \rightarrow \infty} U_{b, t}(x)$, where $U_{b, t}(x)$, $t>b$, is the unique solution of (8.2) satisfying $U_{b, t}(b)=E, U_{b, t}(t)=0$.

It follows as a special case of Theorem 5.1 of this paper that the vector system

$$
\begin{aligned}
u^{\prime \prime} & =A(x) u, \quad 0 \leq x<\infty \\
u(0) & =u_{0}, \quad|u(x)| \text { bounded on } 0 \leq x<\infty,
\end{aligned}
$$

where $A(x)$ is a real symmetric non-negative matrix of functions continuous on $0 \leq x<\infty$, has a unique solution. Moreover, Theorem 6.1 shows that the solution $u(x)$ of (8.3) is the limit, as $N \rightarrow \infty$, of a function $u_{N}(x)$ satisfying $u_{N}^{\prime \prime}=A(x) u_{N}, u_{N}(0)=u_{0}, \mu_{N}(N)=0, N=1,2, \cdots$. In view of the similar characterization of this solution and of the principal solutions described above, it follows that the column vectors of $U(x)$, where $U(x)$ is a principal solution of $U^{\prime \prime}=A(x) U$, are particular bounded solutions of $u^{\prime \prime}=A(x) u$. This fact will be used in the proof of the following lemma.

Lemma 8.1. Suppose $A(x ; h)$ is an $n \times n$ non-negative definite symmetric matrix, continuous jointly in the scalar $x$ and the vector $h$ for $0 \leq x<\infty$ and $h$ in some open set $H$. Let $W_{h}(x)$ be the unique principal solution of

$$
W_{h}^{\prime \prime}(x)=A(x ; h) W_{h}(x),
$$

satisfying

$$
W_{h}(0)=E .
$$

Then, if $h_{0}$ is in $H$ we have $\lim _{h \rightarrow h_{0}} W_{h}(x)=W_{h_{0}}(x)$, uniformly for $x$ on any interval $0 \leq x \leq X$.

We consider the solutions $U=U_{h}(x)$ of the system

$$
\begin{aligned}
U^{\prime \prime} & =A(x ; h) U \\
U(0) & =E, \quad U^{\prime}(0)=E
\end{aligned}
$$

or, equivalently,

$$
\begin{aligned}
U^{\prime} & =V \\
V^{\prime} & =A(x ; h) U \\
U(0) & =E, \quad V(0)=E .
\end{aligned}
$$


The latter is of the form $\left(2.4^{\prime}\right)$ of [6] with $A=0, B=E, C=A(x ; h)$. The solution of (8.4) is non-singular on $0 \leq x<\infty$, since if $\xi$ is a constant vector such that $u=U(x) \xi$ satisfies $u\left(x_{0}\right)=0$ with $x_{0}>0$, then

$$
\begin{aligned}
0 & =\int_{0}^{x_{0}} u^{*}\left(u^{\prime \prime}-A u\right) d x, \\
& =\left.u^{*} u^{\prime}\right|_{0} ^{x_{0}}-\int_{0}^{x_{0}}\left(\left|u^{\prime}\right|^{2}+u^{*} A u\right) d x, \\
& =-|\xi|^{2}-\int_{0}^{x_{0}}\left(\left|u^{\prime}\right|^{2}+u^{*} A u\right) d x,
\end{aligned}
$$

so that $\xi=0$.

Continuing to use the notation of $[6 ; \S 3]$, we compute the value of the constant matrix $\{U, U\}=U^{*}(x) V(x)-V^{*}(x) U(x)$ to be $U^{*}(0) V(0)$ $V^{*}(0) U(0)=0$, and we find that $T=E$. By Theorem 3.1 of [6] we know that any solution $Y(x)$ of $Y^{\prime \prime}=A(x) Y$ with $Y(0)=E$ has the form

$$
Y(x)=U(x)\left[E+\left(\int_{0}^{x} U^{-1}(t) U^{*-1}(t) d t\right) K_{0}\right],
$$

for some constant matrix $K_{0}$.

Now by Theorems 5.1 and 6.1 of [6] we have $W_{h}(x)=\lim _{N \rightarrow \infty} Y_{0 N}(x)$, where $Y_{0 N}^{\prime \prime}=A(x, h) Y_{0 N}, Y_{0 N}(0)=E, Y_{0 N}(N)=0$. But in view of the boundary conditions satisfied by $Y_{0 N}(x)$ we have

$$
Y_{0 N}(x)=U(x)\left[E+\left(\int_{0}^{x} U^{-1}(t) U^{*-1}(t) d t\right) K_{0}\right]
$$

with

$$
K_{0}=-\left(\int_{0}^{N} U^{-1}(t) U^{*-1}(t) d t\right)^{-1}
$$

Hence,

$$
Y_{0 N}(x)=U(x)\left[E-\left(\int_{0}^{x} U^{-1} U^{*-1} d t\right)\left(\int_{0}^{N} U^{-1} U^{*-1} d t\right)^{-1}\right],
$$

and finally,

$$
W_{h}(x)=U_{h}(x)\left[E-\left(\int_{0}^{x} U_{h}^{-1} U_{h}^{*-1} d t\right) M\left(0 ; U_{h}\right)\right] .
$$

We now need an estimate of $U_{h}^{-1}(x) U_{h}^{*-1}(x)$ for large $x$. To this end put $Z_{h}(x)=(1+x)^{-1} U_{h}(x)$. In view of (8.4), one readily verifies that

$$
\left((1+x)^{2} Z_{h}^{\prime}\right)^{\prime}-(1+x)^{2} A(x ; h) Z_{h}=0, \quad Z_{h}(0)=E, \quad Z_{h}^{\prime}(0)=0 .
$$

From this fact it follows that 


$$
\begin{aligned}
0 & =\int_{0}^{x} Z_{h}^{*}\left[\left((1+t)^{2} Z_{h}^{\prime}\right)^{\prime}-(1+t)^{2} A(t ; h) Z_{h}\right] d t \\
& =\left.(1+t)^{2} Z_{h}^{*} Z_{h}^{\prime}\right|_{0} ^{x}-\int_{0}^{x}(1+t)^{2}\left[Z_{h}^{* \prime} Z_{h}^{\prime}+Z_{h}^{*} A(t ; h) Z_{h}\right] d t,
\end{aligned}
$$

and therefore

$$
(1+x)^{2} Z_{h}^{*}(x) Z_{h}^{\prime}(x)=\int_{0}^{x}(1+t)^{2}\left[Z_{h}^{* \prime} Z_{h}^{\prime}+Z_{h}^{*} A Z_{h}\right] d t .
$$

Consequently, $\left(Z_{h}^{*} Z_{h}\right)^{\prime}=Z_{h}^{*} Z_{h}^{\prime}+Z_{h}^{* \prime} Z_{h}=2 Z_{h}^{*} Z_{h}^{\prime} \geq 0$ on $0 \leq x<\infty$, and $Z_{h}^{*}(x) Z_{h}(x) \geq Z_{h}^{*}(0) Z_{h}(0)=E$ for $x \geq 0$; that is, $U_{h}^{*}(x) U_{h}(x) \geq(1+x)^{2} E$ and hence $U_{h}^{-1}(x) U_{h}^{*-1}(x) \leq(1+x)^{-2} E$ on $0 \leq x<\infty$ for $h$ in $H$.

Since as $h \rightarrow h_{0}$ we have $U_{h}(x) \rightarrow U_{h_{0}}(x)$ uniformly on each finite interval $0 \leq x \leq X$, it follows that

$$
\lim _{h \rightarrow h_{0}} M\left(0 ; U_{h}\right)=M\left(0 ; U_{h_{0}}\right) .
$$

This result, with (8.6), proves the lemma.

We can now prove the following theorem:

THEOREM 8.1. If $g_{y y}(x, y)=\left\|g_{y_{i} y_{j}}\right\|$ exists and is continuous for $(x, y)$ in $\Omega: 0 \leq x<\infty,|y|<\infty$, and if $g(x, y)$ satisfies $H_{1}-H_{5}$, then with $y(x ; \alpha)$ as in the beginning of this section, we have that $\partial y(x ; \alpha) / \partial \alpha$, and $\partial y^{\prime}(x ; \alpha) / \partial \alpha$, exist and are continuous in $x, \alpha$ for $0 \leq x<\infty$, $|\alpha|<\infty, j=1,2, \cdots, n$.

Note that if the hypotheses of this theorem are satisfied, then $g_{y y}(x, y) \geq 0$ for $(x, y)$ in $\Omega$. We denote by $e^{(j)}$ the unit vector having all components zero but the $j$ th, and we let $\Delta \alpha=e^{(j)} h, \Delta y=y(x ; \alpha+\Delta \alpha)-$ $y(x ; \alpha)$, where $h$ is a real scalar. Then

$$
\begin{aligned}
(\Delta y)^{\prime \prime} & =g_{y}(x, y(x ; \alpha+\Delta \alpha))-g_{y}(x, y(x ; \alpha)) \\
& =\left(\int_{0}^{1} g_{y y}(x, y(x ; \alpha)+\theta \Delta y) d \theta\right) \Delta y,
\end{aligned}
$$

so that

$$
\left(\frac{\Delta y}{h}\right)^{\prime \prime}=\left(\int_{0}^{1} g_{y y}(x, y(x ; \alpha)+\theta \Delta y) d \theta\right)\left(\frac{\Delta y}{h}\right), \quad h \neq 0 .
$$

In Lemma 8.1 we identity $A(x ; h)$ as $\int_{0}^{1} g_{y y}(x, y(x ; \alpha)+\theta \Delta y) d \theta$, where $\alpha$ is fixed, and we identify $h_{0}$ as zero. We note that

$$
\left(\frac{\Delta y}{h}\right)_{x=0}=e^{(j)} \text {, and }\left|\frac{\Delta y(x)}{h}\right|=\frac{|\Delta y(x)|}{|h|} \leq \frac{|\Delta y(0)|}{|h|} \leq 1 \text {, }
$$

$0 \leq x<\infty$. Hence, $(1 / h) \Delta y$ is the unique bounded solution of $z^{\prime \prime}=A(x ; h) z$ satisfying $z(0)=e^{(j)}$. As explained above, the unique principal solution of 


$$
Z_{l b}^{\prime \prime}=A(x ; h) Z_{h},
$$

satisfying

$$
Z_{h}(0)=E,
$$

is the same as the bounded solution of $\left(8.7^{\prime}\right)$, (8.7') guaranteed by Theorem 6.1 of this paper, of which $(1 / h) \Delta y$ is the $j$ th column vector, for $h \neq 0$. Lemma 8.1 then implies that $\lim _{h \rightarrow 0}(1 / h) \Delta y(x)$ exists and is equal to the $j$ th column vector of $Z_{0}(x)$; that is, for all $\alpha$, the vector function $y_{\alpha_{j}}(x ; \alpha)=\left(\partial / \partial \alpha_{j}\right) y(x ; \alpha)$ exists and satisfies

$$
\left(y_{\alpha_{j}}(x ; \alpha)\right)^{\prime \prime}=g_{y y}(x, y(x ; \alpha)) y_{x_{j}}(x ; \alpha) ; \quad 0 \leq x<\infty .
$$

Since $\left|y_{\alpha_{j}}(x ; \alpha)\right| \leq 1$, we may use Lemma 8.1 with $h=\alpha$ in conjunction with the inequality

$$
\left|y_{\alpha_{j}}(\bar{x}, \bar{\alpha})-y_{\alpha_{j}}(x, \alpha)\right| \leq\left|y_{\alpha_{j}}(\bar{x} ; \bar{\alpha})-y_{\alpha_{j}}(\bar{x} ; \alpha)\right|+\left|y_{\alpha_{j}}(\bar{x} ; \alpha)-y_{\alpha_{j}}(x ; \alpha)\right|
$$

to show that $y_{\gamma_{j}}(x ; \alpha)$ is continuous in $x$ and $\alpha$. Differentiation of the right hand member of (8.1) with respect to $\alpha_{j}$ shows the existence of $\left(\partial / \partial \alpha_{j}\right) y^{\prime}(x ; \alpha)$ and its continuity with respect to $x$ and $\alpha$.

\section{BIBLIOGRAPHY}

1. R. Bellman, Stability theory of differential equations, McGraw-Hill, New York, 1953.

2. G. H. Hardy, J. E. Littlewood and G. Pólya, Inequalities, 2nd ed., Cambridge University Press, London, 1952.

3. E. Hille, Non-oscillation theorems, A.M.S. Trans., 64 (1948), 234-252.

4. E. L. Ince, Ordinary differential equations, Longmans, Green and Co. Ltd., London, 1927.

5. W. T. Reid, Oscillation criteria for linear differential systems with complex coefficients, Pacific J. Math., 6 (1956), 733-751.

6. - Principal solutions of non-oscillatory self-adjoint linear differential systems, Pacific J. Math., 8 (1958), 147-169.

7. F. Riesz and B. Sz.-Nagy, Leçons d'Analyse fonctionnelle, 2d ed., Budapest, 1953.

8. G. Sansone, Equazioni differenziali nel Campo reale, part 2, 2d ed., Bolonga, 1949.

LOYOLA UNIVERSITY

ChICAgo 



\title{
PACIFIC JOURNAL OF MATHEMATICS
}

\section{EDITORS}

\author{
David Gilbarg \\ itanford University \\ itanford, California \\ F. H. Brownele \\ Jniversity of Washington \\ ieattle 5 , Washington
}

\author{
A. L. Whiteman
}

University of Southern California Los Angeles 7. California

L. J. Paige

University of California

Los Angeles 24, California

ASSOCIATE EDITORS
ミ. F. BECKENBACH
E. HEWITT
M. OHTSUKA
E. SPANIER
r. M. CHERRY
A. HORN
H. L. ROYDEN
E. G. STRAUS
). DERRY
L. NACHBIN
M. M. SCHIFFER
F. WOLF

\section{SUPPORTING INSTITUTIONS}

JNIVERSITY OF BRITISH COLUMBIA
ZALIFORNIA INSTITUTE OF TECHNOLOGY
JNIVERSITY OF CALIFORNIA
UONTANA STATE UNIVERSITY
JNIVERSITY OF NEVADA
NEW MEXICO STATE UNIVERSITY
JREGON STATE COLLEGE
JNIVERSITY OF OREGON
JSAKA UNIVERSITY
JNIVERSITY OF SOUTHERN CALIFORNIA

STANFORD UNIVERSITY

UNIVERSITY OF TOKYO

UNIVERSITY OF UTAH

WASHINGTON STATE COLLEGE

UNIVERSITY OF WASHINGTON

AMERICAN MATHEMATICAL SOCIETY CALIFORNIA RESEARCH CORPORATION HUGHES AIRCRAFT COMPANY

SPACE TECHNOLOGY LABORATORIES

NAVAL ORDNANCE TEST STATION

Printed in Japan by Kokusai Bunken Insatsusha

(International Academic Printing Co., Ltd.), Tokyo, Japan 


\section{Pacific Journal of Mathematics}

\section{Vol. 10, No. $1 \quad$ September, 1960}

Richard Arens, Extensions of Banach algebras................... 1

Fred Guenther Brauer, Spectral theory for linear systems of differential equations....................................... 17

Herbert Busemann and Ernst Gabor Straus, Area and normality ......... 35

J. H. Case and Richard Eliot Chamberlin, Characterizations of tree-like

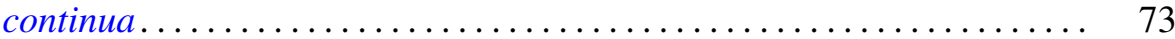

Ralph Boyett Crouch, Characteristic subgroups of monomial groups...... 85

Richard J. Driscoll, Existence theorems for certain classes of two-point boundary problems by variational methods ................. 91

A. M. Duguid, A class of hyper-FC-groups ................. 117

Adriano Mario Garsia, The calculation of conformal parameters for some

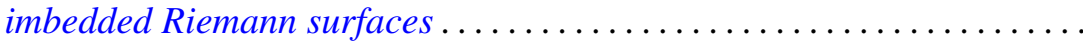

Irving Leonard Glicksberg, Homomorphisms of certain algebras of measures ........................................... 167

Branko Grünbaum, Some applications of expansion constants . . . . . . . . . 193

John Hilzman, Error bounds for an approximate solution to the Volterra integral equation ................................... 203

Charles Ray Hobby, The Frattini subgroup of a p-group ............... 209

Milton Lees, von Newmann difference approximation to hyperbolic

equations...................................... 213

Azriel Lévy, Axiom schemata of strong infinity in axiomatic set theory .... 223

Benjamin Muckenhoupt, On certain singular integrals ............... 239

Kotaro Oikawa, On the stability of boundary components ............. 263

J. Marshall Osborn, Loops with the weak inverse property .............. 295

Paulo Ribenboim, Un théorème de réalisation de groupes réticulés ....... 305

Daniel Saltz, An inversion theorem for Laplace-Stieltjes transforms ....... 309

Berthold Schweizer and Abe Sklar, Statistical metric spaces ........... 313

Morris Weisfeld, On derivations in division rings ................ 335

Bertram Yood, Faithful ${ }^{*}$-representations of normed algebras ........... 345 